\title{
Lateral Vibrations of a Cable-Stayed Bridge under Crowd Excitation
}

\author{
Lijun Ouyang, ${ }^{1}$ Caihong Wang, ${ }^{2}$ Bin Zhen, ${ }^{1}$ and Jian $\mathrm{Xu}^{3}$ \\ ${ }^{1}$ School of Environment and Architecture, University of Shanghai for Science and Technology, Shanghai 200093, China \\ ${ }^{2}$ Shanghai Publishing and Printing College, Shanghai Publication and Media Research Institute, Shanghai 200092, China \\ ${ }^{3}$ School of Aerospace Engineering and Mechanics, Tongji University, Shanghai 200092, China \\ Correspondence should be addressed to Bin Zhen; zhenbin80@163.com
}

Received 13 June 2015; Revised 29 August 2015; Accepted 30 August 2015

Academic Editor: Stefano Lenci

Copyright (C) 2015 Lijun Ouyang et al. This is an open access article distributed under the Creative Commons Attribution License, which permits unrestricted use, distribution, and reproduction in any medium, provided the original work is properly cited.

A cable-stayed bridge model under crowd excitation is established in this paper by considering the geometric nonlinear property of the cables. Lateral vibrations of the model are investigated by employing the center manifold theory, and the first-order approximation solution of the periodic vibration of the bridge is derived by using the energy method. Numerical simulations are carried out to verify the validity of our analytical expressions. Our research shows that the existence of the cables can reduce the amplitude and frequency of the bridge, especially for the large amplitude case. This might explain why measured data of a cable-stayed bridge (T-bridge in Japan) vibrating under crowd excitation are much less than the theoretical results reported in previous studies in which the cable-stayed bridge is viewed as a single-degree-of-freedom system. Our analysis results suggest that the structure types of footbridges should not be easily ignored in the study of pedestrian-footbridge interaction.

\section{Introduction}

In the last decade attention has been focused on the lateral vibrations of footbridges induced by pedestrians since the London Millennium Bridge was opened on 10 June 2000 $[1,2]$. After that, more and more excessive lateral vibrations of footbridges in various structures have been reported, including suspension bridges [3, 4], arch bridges, truss bridges [1], and cable-stayed bridges [5]. It seems that the occurrence of excessive lateral vibrations of a footbridge is independent of its structure type.

The mechanisms of the lateral vibrations of footbridges exerted by pedestrians have been paid lots of attention in recent ten years. Plentiful of experiments suggested that the lateral vibrations of footbridges are caused by the zigzag movements of pedestrians. When pedestrians on a footbridge step with their right and left feet in turn, the gravity center of their bodies has a lateral oscillation, which induces lateral dynamic time-varying forces on the surface of the bridge. As a consequence the bridge begins to sway laterally. The pedestrians instinctively feel more comfortable to walk in synchronization with the lateral motion of the bridge, which further increases its response. The frequency of the lateral force induced by pedestrians is about between 0.7 and $1.2 \mathrm{~Hz}$ [6]. If the natural frequency of the bridge is within the range of the lateral walking frequency, direct resonance between pedestrians and the footbridge may be achieved [5] If there exists a $2: 1$ ratio between the vertical and lateral mode frequencies of the bridge, internal resonance $[3,7]$ is possible. The energy may flow from the vertical mode to the lateral mode due to structural nonlinearities. Dynamic interaction mechanisms have attracted much interests in past decade because they allow the research for the critical number of pedestrians. A lot of dynamic models describing the interaction between pedestrians and a footbridge have been proposed to understand the occurrence of excessive lateral vibrations of the footbridge [1, 4, 7-11]. To explain the occurrence of lateral vibrations with $0.48 \mathrm{~Hz}$ in the Millennium Bridge, Piccardo and Tubino [12] proposed the parametric excitation mechanism by assuming that the lateral pedestrian-induced force is proportional to the lateral bridge displacement. The above research for dynamic interaction suggested that the key to lateral vibration problem mainly lies in the measurement and/or modeling of the lateral 
force exerted by pedestrians on footbridges. However, the force exerted by pedestrians is generally tackled by using an empirical approach; strict and exact mathematical models are not available yet.

On the other side, in almost all dynamic models proposed to describe the interaction between pedestrians and a footbridge, the footbridge always is simplified as a singledegree-freedom system. On an intuitive level, such simplification may not be rational to all types of footbridges, for example, suspension bridges and cable-stayed bridges. In fact, McRobie et al. [13] experimentally investigated the "lock-in" phenomenon by constructing a section model with cables and bridge deck. Zhou and Ji [14] theoretically and experimentally analyzed a generalized suspension system developed from the section model. Their research showed that cables have great influence on free vibrations of the suspension system, whereas the dynamic behaviour of the suspension system under crowd excitation has not been considered. Cablestayed bridges are important engineering structures widely applied all over the world. A cable-stayed bridge consists of a deck suspended from stay cables and supported on piers and towers. Geurts et al. [15] reported that Erasmus bridge in Netherlands, opened to the public for just two months in 1996, had to be closed because of the large amplitude vibrations of the cables. Lilien and Pinto Da Costa [16] found that a dynamic instability of the cables may occur due to small periodic movements of the girder and the masts. Fujino et al. [5] argued that cables may have intensive vibrations induced by deck when the natural frequency of the cables approximates to half natural frequency of the deck. Nevertheless, seldom theoretical analysis for cablestayed bridges with nonlinear couples between cables and deck under crowd excitation has been carried out.

The paper focuses on the lateral vibrations of a cablestayed bridge under crowd excitation. The cable-stayed bridge model consists of cables and deck which are nonlinearly coupled. Nakamura's assumption [4] is adopted to model the lateral force induced by crowd excitation. We will pull all our emphasis on whether and how the cables affect the lateral vibrations of the deck under crowd excitation. The rest of the paper is organized as follows: the governing equation of the pedestrian-bridge system is established in Section 2; the qualitative and quantitative analysis for the governing equation are presented in Sections 3 and 4 respectively; numerical verifications and discussion are demonstrated in Section 5; conclusions are drawn in Section 6.

\section{The Governing Equation}

The cable-stayed bridge model investigated in this paper is illustrated in Figure 1, in which line $A B$ represents cables and the rigid block of the deck. Line $A B$ has a length $L$, mass $m_{1}$, stiffness $E A$, tension $T_{0}$, and damping coefficient $c_{1}$. For simplicity all the matters in line $A B$ are assumed to be together in the center of mass (point $C$ ). The rigid block has a mass $m_{2}$, stiffness $k$, and damping coefficient $c_{2}$. Assume that the lateral displacements of point $C$ and the rigid block are $x_{1}$ and $x_{2}$, respectively. $\dot{x}_{1,2}, \ddot{x}_{1,2}$ represent corresponding

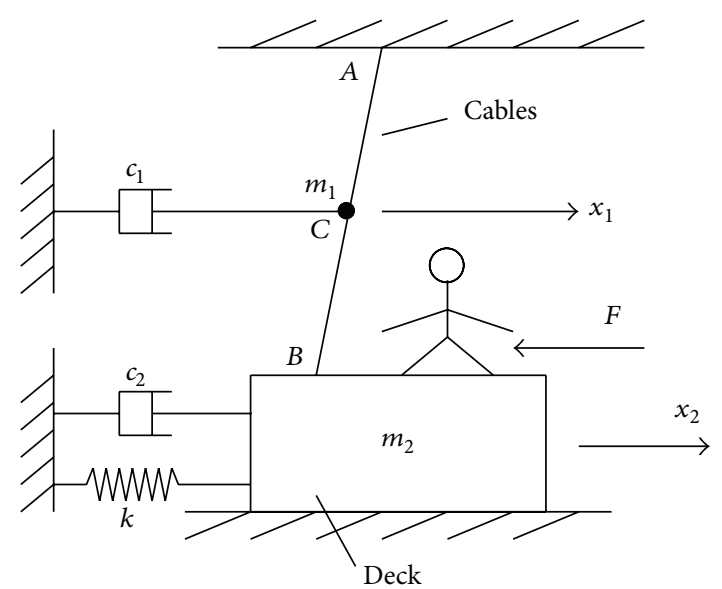

Figure 1: The cable-stayed bridge diagram.

velocities and accelerations, respectively. The lateral force induced by pedestrians on the bridge is denoted by $F$.

According to Nakamura's assumption [4], $F$ is given by

$$
F=k_{1} k_{2} M_{p} g G(f) H\left(\dot{x}_{2}\right)
$$

where coefficient $k_{1}$ is the ratio of lateral force to pedestrians' weight; coefficient $k_{2}$ is the percentage of pedestrians who synchronize to bridge vibration; $M_{p} g$ is modal self-weight of pedestrians; $G(f)$ is a function describing how pedestrians synchronize with the natural frequency of a bridge; and $H\left(\dot{x}_{2}\right)$ is a function that describes the pedestrians' synchronization nature. Nakamura assumed that the pedestrians synchronize proportionally with the deck velocity $\dot{x}_{2}$ at low velocities. When the deck velocity becomes large, pedestrians feel uncomfortable and then they decrease their walking pace which limits the deck response at a certain level rather than infinite one. Based on the analysis of Zhen et al. [17], $H\left(\dot{x}_{2}\right)$ can be expressed as

$$
H\left(\dot{x}_{2}\right)=\tanh \left(k_{3} \dot{x}_{2}\right)
$$

in which the coefficient $k_{3}$ indicates the saturation rate of the pedestrian-induced force.

By applying Newton's second law of motion and considering the geometric nonlinearity of the cables, the governing equation of the cable-stayed bridge under crowd excitation is derived as follows:

$$
\begin{aligned}
& \ddot{x}_{1}+\alpha_{1} \dot{x}_{1}+\beta_{1} x_{1}+\gamma_{1}\left[x_{1}^{3}+\left(x_{1}-x_{2}\right)^{3}\right]=0, \\
& \ddot{x}_{2}+\alpha_{2} \dot{x}_{2}+\beta_{2} x_{2}+\gamma_{2}\left(x_{1}-x_{2}\right)^{3}=\sigma \tanh \left(k_{3} \dot{x}_{2}\right),
\end{aligned}
$$

where $\alpha_{1}=c_{1} / m_{1}, \alpha_{2}=c_{2} / m_{2}, \beta_{1}=2 T_{0} / m_{1} L, \beta_{2}=\mathrm{k} / \mathrm{m}_{2}$, $\gamma_{1}=E A / 2 m_{1} L^{3}, \gamma_{2}=E A / 2 m_{2} L^{3}$, and $\sigma=\left(k_{1} k_{2} G(f) M_{p} g\right) /$ $m_{2}$.

According to the physics meanings, $\alpha_{1,2}, \beta_{1,2}, \gamma_{1,2}>0$. Furthermore, we assume that $\beta_{2}>\gamma_{2}$ in the following analysis. 


\section{Qualitative Analysis for the Cable-Stayed Bridge Model}

By letting

$$
\begin{aligned}
& x_{1}=y_{1}, \\
& \dot{x}_{1}=y_{2}, \\
& x_{2}=y_{3}, \\
& \dot{x}_{2}=y_{4},
\end{aligned}
$$

(3) can be rewritten as

$$
\begin{aligned}
& \dot{y}_{1}=y_{2}, \\
& \dot{y}_{2}=-\beta_{1} y_{1}-\alpha_{1} y_{2}-\gamma_{1}\left[y_{1}^{3}+\left(y_{1}-y_{3}\right)^{3}\right], \\
& \dot{y}_{3}=y_{4}, \\
& \dot{y}_{4}=-\beta_{2} y_{3}-\alpha_{2} y_{4}+\sigma \tanh \left(k_{3} y_{4}\right)-\gamma_{2}\left(y_{1}-y_{3}\right)^{3} .
\end{aligned}
$$

Obviously, $(0,0,0,0)$ is an equilibrium of $(5)$. The characteristic equation of (5) near the origin is

$$
\begin{gathered}
\lambda^{4}+\left(\alpha_{1}-\delta\right) \lambda^{3}+\left(\beta_{1}+\beta_{2}-\alpha_{1} \delta\right) \lambda^{2} \\
+\left(\alpha_{1} \beta_{2}-\delta \beta_{1}\right) \lambda+\beta_{1} \beta_{2}=0
\end{gathered}
$$

where $\delta=\sigma k_{3}-\alpha_{2}$. Since $\alpha_{i}, \beta_{i}, \gamma_{i}>0, i=1,2$, it is easy to verify that (5) has a stable origin if $\delta<0$ and an unstable one if $\delta>0$. When $\delta=0$ (6) has a pair of purely imaginary roots: $\lambda_{1,2}= \pm i \sqrt{\beta_{2}}$. Regarding $\lambda$ as a function of $\delta$ in (6) and taking the derivative of $\lambda$ with respect to $\delta$, one has

$$
\begin{aligned}
& \frac{d \lambda}{d \delta} \\
& =\frac{\lambda\left(\lambda^{2}+\alpha_{1} \lambda+\beta_{1}\right)}{4 \lambda^{3}-3\left(\delta-\alpha_{1}\right) \lambda^{2}-2\left(\delta \alpha_{1}-\beta_{1}-\beta_{2}\right) \lambda-\beta_{1} \delta+\alpha_{1} \beta_{2}} .
\end{aligned}
$$

Substituting $\lambda= \pm i \sqrt{\beta_{2}}$ and $\delta=0$ into (7) yields

$$
\left(\frac{d \lambda}{d \delta}\right)_{\delta=0}=\frac{1}{2}>0 .
$$

According to Hopf bifurcation theory, system (5) undergoes a Hopf bifurcation near $\delta=0$, and a limit cycle appears when $\delta>0$. This means that periodic vibrations occur in the cable-stayed bridge model under crowd excitation. To further determine the stability of the limit cycle, we employ center manifold theory to analyze system (5).

If $\delta=0,(6)$ has a pair of purely imaginary roots and two negative real roots, which satisfies the condition for center manifold theory. Assume that a local center manifold can be expressed by

$$
\begin{aligned}
& y_{1}=h_{1}\left(y_{3}, y_{4}\right), \\
& y_{2}=h_{2}\left(y_{3}, y_{4}\right),
\end{aligned}
$$

where $h_{1}$ and $h_{2}$ are vector functions of nonlinear terms of $y_{3}$ and $y_{4}$. Assume that $y_{1}$ and $y_{2}$ can be transformed into power series

$$
\begin{aligned}
y_{1}= & h_{10} y_{3}^{3}+h_{11} y_{3}^{2} y_{4}+h_{12} y_{3} y_{4}^{2}+h_{13} y_{4}^{3} \\
& +O\left(y_{3}, y_{4}\right), \\
y_{2}= & h_{20} y_{3}^{3}+h_{21} y_{3}^{2} y_{4}+h_{22} y_{3} y_{4}^{2}+h_{23} y_{4}^{3} \\
& +O\left(y_{3}, y_{4}\right),
\end{aligned}
$$

where $h_{1 i}$ and $h_{2 i}(i=0,1,2,3, \ldots)$ are coefficients to be determined. $O(\cdot)$ represents the higher orders of $y_{3,4}$. Substituting (10) into (5) and setting the coefficients of each power of $y_{3,4}$ equal to zero would yield the following set of equations:

$$
\begin{aligned}
h_{10} & =\frac{1}{A}\left[\left(7 \beta_{1} \beta_{2}-9 \beta_{2}^{2}\right) \gamma_{1} \alpha_{1}^{2}\right. \\
& \left.+\gamma_{1}\left(\beta_{1}^{3}-17 \beta_{1}^{2} \beta_{2}+79 \beta_{1} \beta_{2}^{2}-63 \beta_{2}^{3}\right)\right], \\
h_{11} & =\frac{1}{A}\left(3 \beta_{2} \alpha_{1}^{2}+\beta_{1}^{2}-6 \beta_{1} \beta_{2}+21 \beta_{2}^{2}\right), \\
h_{12} & =\frac{6 \gamma_{1}}{A}\left(\beta_{1} \alpha_{1}^{2}-\beta_{1}^{2}+10 \beta_{1} \beta_{2}-9 \beta_{2}^{2}\right), \\
h_{13} & =-\frac{6 \gamma_{1} \alpha_{1}}{A}\left(\alpha_{1}^{2}-2 \beta_{1}+10 \beta_{2}\right),
\end{aligned}
$$

where

$$
\begin{aligned}
A= & 9 \beta_{2}^{2} \alpha_{1}^{4}+2\left(-18 \beta_{1} \beta_{2}^{2}+10 \beta_{1}^{2} \beta_{2}+90 \beta_{2}^{3}\right) \alpha_{1}^{2} \beta_{4} \\
& -20 \beta_{1}^{3} \beta_{2}+118 \beta_{1}^{2} \beta_{2}^{2}-180 \beta_{1} \beta_{2}^{3}+81 \beta_{2}^{4} .
\end{aligned}
$$

Then the control equations on the center manifold are given by

$$
\begin{aligned}
\dot{y}_{3}= & y_{4}, \\
\dot{y}_{4}= & -\beta_{2} y_{3}-\alpha_{2} y_{4}+\sigma \tanh k_{3} y_{4} \\
& -\gamma_{2}\left[h_{1}\left(y_{3}, y_{4}\right)-y_{3}\right]^{3} .
\end{aligned}
$$

By letting

$$
\begin{aligned}
& u=y_{3}, \\
& v=-\frac{1}{\sqrt{\beta_{2}}} y_{4},
\end{aligned}
$$

(13) can be rewritten as

$$
\begin{aligned}
& \dot{u}=-\sqrt{\beta_{2}} v, \\
& \dot{v}=\sqrt{\beta_{2}} u+Q,
\end{aligned}
$$

where

$$
Q=-\frac{\gamma_{2}}{\sqrt{\beta_{2}}} u^{3}-\frac{1}{3} \beta_{2} \sigma^{3} v^{3}+O\left(u^{3}, v^{3}\right) .
$$


When $\delta=\sigma k_{3}-\alpha_{2}=0$, the first Lyapunov coefficient of system (13) can be calculated

$$
\begin{aligned}
l_{1}= & \frac{1}{16}\left(\frac{\partial^{3} F}{\partial u^{2} \partial v}+\frac{\partial^{3} F}{\partial v^{3}}\right) \\
& -\frac{1}{16 \sqrt{\beta_{2}}}\left[\frac{\partial^{2} F}{\partial u \partial v}\left(\frac{\partial^{2} F}{\partial u^{2}}+\frac{\partial^{2} F}{\partial v^{2}}\right)\right] \\
= & -\frac{1}{8} \beta_{2}\left(\frac{\alpha_{2}}{k_{3}}\right)^{3}<0 .
\end{aligned}
$$

The first Lyapunov coefficient is less than zero, which means that original system (5) undergoes a Hopf bifurcation near $\delta=0$ and a stable limit cycle occurs. To discuss the dynamic response of cables and its influence on the deck in greater detail, we will analytically calculate the periodic solution in system (3) in next section.

\section{Quantitative Analysis for the Cables-Stayed Bridge Model}

In this section we use the energy method [18] to calculate the periodic solution of (3).

(1) Denote

$$
\begin{aligned}
g_{1}\left(x_{1}\right)= & \beta_{1} x_{1}+2 \gamma_{1} x_{1}^{3}, \\
g_{2}\left(x_{2}\right)= & \beta_{2} x_{2}-\gamma_{2} x_{2}^{3}, \\
f_{1}(x, \dot{x})= & \alpha_{1} \dot{x}_{1}+\gamma_{1}\left(3 x_{1} x_{2}^{2}-3 x_{1}^{2} x_{2}-x_{2}^{3}\right), \\
f_{2}(x, \dot{x})= & \alpha_{2} \dot{x}_{2}-\sigma \tanh \left(k_{3} \dot{x}_{2}\right) \\
& +\gamma_{2}\left(x_{1}^{3}-3 x_{1}^{2} x_{2}+3 x_{1} x_{2}^{2}\right) .
\end{aligned}
$$

Considering the physics meanings of parameters in (3) and assuming that the vibration amplitudes are not too large $\left(\left|x_{1,2}\right|<1 m\right)$, one has

$$
\begin{aligned}
& x_{1} g_{1}\left(x_{1}\right)_{x_{1} \neq 0}=\beta_{1} x_{1}^{2}+2 \gamma_{1} x_{1}^{4}>0, \\
& x_{2} g_{2}\left(x_{2}\right)_{x_{2} \neq 0}=\beta_{2} x_{2}^{2}-\gamma_{2} x_{2}^{4}>0 .
\end{aligned}
$$

Therefore, the energy method can be applied to (3) to calculate its approximation periodic solution.

(2) The potential energy functions of system (3) can be expressed by

$$
\begin{aligned}
V_{1}\left(x_{1}\right) & =\int_{0}^{x_{1}}\left(\beta_{1} x_{1}+2 \gamma_{1} x_{1}^{3}\right) d x_{1} \\
& =\frac{1}{2}\left(\beta_{1} x_{1}^{2}+\gamma_{1} x_{1}^{4}\right) \\
V_{2}\left(x_{2}\right) & =\int_{0}^{x_{2}}\left(\beta_{2} x_{2}-\gamma_{2} x_{2}^{3}\right) d x_{2} \\
& =\frac{1}{4}\left(2 \beta_{2} x_{2}^{2}-\gamma_{2} x_{2}^{4}\right) .
\end{aligned}
$$

$V_{i}(x), i=1,2$, are even functions; thus $b_{1,2}=0$ hold [18]. The coordinate change formulas can be written as

$$
\begin{aligned}
x_{i} & =a_{i} \cos \left(\theta_{i}\right), \quad(i=1,2), \\
\dot{x}_{1} & = \pm \sqrt{2\left(V_{1}\left(a_{1}\right)-V_{1}\left(a_{1} \cos \left(\theta_{1}\right)\right)\right)} \\
& = \pm a_{1}\left|\sin \left(\theta_{1}\right)\right| \sqrt{\beta_{1}+\frac{3}{2} \gamma_{1} a_{1}^{2}+\frac{1}{2} \gamma_{1} a_{1}^{2} \cos \left(2 \theta_{1}\right)}, \\
\dot{x}_{2} & = \pm \sqrt{2\left(V_{2}\left(a_{2}\right)-V_{2}\left(a_{2} \cos \left(\theta_{2}\right)\right)\right)} \\
& = \pm a_{2}\left|\sin \left(\theta_{2}\right)\right| \sqrt{\beta_{2}-\frac{3}{4} \gamma_{2} a_{2}^{2}-\frac{1}{4} \gamma_{2} a_{2}^{2} \cos \left(2 \theta_{2}\right)} .
\end{aligned}
$$

Ignoring the terms of the harmonic expansions higher than the second, the coordinate change formulas can be simplified as

$$
\begin{aligned}
& \dot{x}_{1}=-a_{1} \sin \left(\theta_{1}\right) A_{1}\left(1+A_{2} \cos \left(2 \theta_{1}\right)\right), \\
& \dot{x}_{2}=-a_{2} \sin \left(\theta_{2}\right) B_{1}\left(1+B_{2} \cos \left(2 \theta_{2}\right)\right),
\end{aligned}
$$

where

$$
\begin{aligned}
A_{1} & =\sqrt{\beta_{1}+\frac{3}{2} \gamma_{1} a_{1}^{2}}>0, \\
0 & <A_{2}=\frac{\gamma_{1} a_{1}^{2}}{4 \beta_{1}+6 \gamma_{1} a_{1}^{2}}<1, \\
B_{1} & =\sqrt{\beta_{2}-\frac{3}{4} \gamma_{2} a_{2}^{2}}>0, \\
-1 & <B_{2}=-\frac{\gamma_{2} a_{2}^{2}}{8 \beta_{2}-6 \gamma_{2} a_{2}^{2}}<0 .
\end{aligned}
$$

(3) Denote the energy of $m_{1}$ and $m_{2}$ by $E_{1}$ and $E_{2}$, respectively. $d E_{1} / d t$ and $d E_{2} / d t$ can be calculated, in which the terms of the harmonic expansions higher than the second will be ignored:

$$
\begin{aligned}
\frac{d E_{1}}{d t}= & -f_{1}(x, \dot{x}) \dot{x}_{1} \\
= & C_{1}+C_{2} \cos \left(2 \theta_{1}\right) \\
& +C_{3}\left[\sin \left(\theta_{1}-\theta_{2}\right)+\sin \left(\theta_{1}+\theta_{2}\right)\right] \\
& +C_{4} \sin \left(2 \theta_{1}-2 \theta_{2}\right)+C_{5} \sin \left(3 \theta_{1}-3 \theta_{2}\right) \\
& +C_{6} \sin \left(2 \theta_{1}\right)+C_{7} \sin \left(3 \theta_{1}-\theta_{2}\right) \\
& +C_{8} \sin \left(\theta_{1}-3 \theta_{2}\right)+C_{9} \sin \left(4 \theta_{1}-2 \theta_{2}\right) \\
\triangleq & F_{1}(E, \theta),
\end{aligned}
$$




$$
\begin{aligned}
\frac{d E_{2}}{d t}= & -f_{2}(x, \dot{x}) \dot{x}_{2} \\
= & D_{1}+D_{2} \cos \left(2 \theta_{2}\right) \\
& +D_{3}\left[\sin \left(\theta_{1}-\theta_{2}\right)-\sin \left(\theta_{1}+\theta_{2}\right)\right] \\
& +D_{4} \sin \left(2 \theta_{1}-2 \theta_{2}\right)+D_{5} \sin \left(3 \theta_{1}-3 \theta_{2}\right) \\
& +D_{6} \sin \left(2 \theta_{2}\right)+D_{7} \sin \left(3 \theta_{1}-\theta_{2}\right) \\
& +D_{8} \sin \left(\theta_{1}-3 \theta_{2}\right)+D_{9} \sin \left(2 \theta_{1}-4 \theta_{2}\right) \\
\triangleq & F_{2}(E, \theta),
\end{aligned}
$$

where

$$
\begin{aligned}
C_{1} & =\frac{1}{4}\left(2 A_{2}-2-A_{2}^{2}\right) A_{1}^{2} a_{1}^{2} \alpha_{1}, \\
C_{2} & =\frac{1}{8}\left(4+3 A_{2}^{2}-8 A_{2}\right) A_{1}^{2} \alpha_{1} a_{1}^{2}, \\
C_{3} & =-\frac{3}{8} \gamma_{1} a_{1}^{3} A_{1} a_{2}+\frac{3}{16} \gamma_{1}\left(A_{2}-2\right) A_{1} a_{2}^{3} a_{1}, \\
C_{4} & =\frac{3}{8} \gamma_{1} a_{1}^{2} A_{1} a_{2}^{2}, \\
C_{5} & =-\frac{1}{16} \gamma_{1} a_{1} A_{1} a_{2}^{3} A_{2}, \\
C_{6} & =\frac{3}{4} \gamma_{1} a_{1}^{2} A_{1} a_{2}^{2}, \\
C_{7} & =-\frac{3}{16} \gamma_{1}\left(A_{2}+2\right) A_{1} a_{2} a_{1}^{3}-\frac{3}{16} \gamma_{1} a_{1} A_{1} a_{2}^{3} A_{2}, \\
C_{8} & =\frac{1}{16} \gamma_{1}\left(A_{2}-2\right) A_{1} a_{2}^{3} a_{1}, \\
C_{9} & =\frac{3}{16} \gamma_{1} a_{1}^{2} A_{1} a_{2}^{2} A_{2}, \\
D_{1} & =\frac{B_{1}^{2} a_{2}^{2}}{24}\left[\left(8 B_{2}-3\right) B_{1}^{2} a_{2}^{2} k_{3}^{3} \sigma\right. \\
& \left.-12\left(B_{2}-1\right)\left(k_{3} \sigma-\alpha_{2}\right)\right],
\end{aligned}
$$$$
D_{2}=\frac{1}{12}\left(2-7 B_{2}\right) k_{3}^{3} B_{1}^{4} a_{2}^{4} \sigma+\frac{1}{8}\left(8 B_{2}-4\right) a_{2}^{2} B_{1}^{2}\left(k_{3} \sigma\right.
$$$$
\left.-\alpha_{2}\right) \text {, }
$$$$
D_{3}=\frac{1}{16}\left(\left(-6+3 B_{2}\right) a_{2} a_{1}^{3}-6 a_{2}^{3} a_{1}\right) \gamma_{2} B_{1} \text {, }
$$$$
D_{4}=\frac{3}{8} \gamma_{2} a_{2}^{2} B_{1} a_{1}^{2} \text {, }
$$$$
D_{5}=-\frac{1}{16} \gamma_{2} a_{2} B_{1} a_{1}^{3} B_{2}
$$$$
D_{6}=-\frac{3}{4} \gamma_{2} a_{2}^{2} B_{1} a_{1}^{2} \text {, }
$$

$$
\begin{aligned}
& D_{7}=\frac{1}{16}\left(B_{2}-2\right) a_{2} a_{1}^{3} \gamma_{2} B_{1}, \\
& D_{8}=-\frac{1}{16}\left(3 a_{2} a_{1}^{3} B_{2}+\left(6+3 B_{2}\right) a_{2}^{3} a_{1}\right) \gamma_{2} B_{1}, \\
& D_{9}=\frac{3}{16} \gamma_{2} a_{2}^{2} B_{1} a_{1}^{2} B_{2} .
\end{aligned}
$$

(4) $d \theta_{1} / d t$ and $d \theta_{2} / d t$ are given by

$$
\begin{aligned}
& \frac{d \theta_{1}}{d t}=\frac{1}{a_{1}^{2}\left(\beta_{1}+2 \gamma_{1} a_{1}^{2}\right)}\left[H_{1}\left(1+\cos \left(2 \theta_{2}\right)\right)\right. \\
& +H_{2} \sin \left(2 \theta_{1}\right)+H_{3} \cos \left(2 \theta_{1}\right)
\end{aligned}
$$$$
+H_{4}\left(\cos \left(\theta_{1}+\theta_{2}\right)+\cos \left(\theta_{1}-\theta_{2}\right)\right)
$$$$
+H_{5} \cos \left(2 \theta_{1}-2 \theta_{2}\right)+H_{6} \cos \left(3 \theta_{1}-3 \theta_{2}\right)
$$$$
+H_{7} \cos \left(\theta_{1}-3 \theta_{2}\right)+H_{8} \cos \left(3 \theta_{1}-\theta_{2}\right)
$$$$
\left.+H_{9} \cos \left(4 \theta_{1}-2 \theta_{2}\right)\right]+A_{1}\left(1+A_{2} \cos \left(2 \theta_{1}\right)\right) \text {, }
$$$$
\frac{d \theta_{2}}{d t}=\frac{1}{a_{2}^{2}\left(\beta_{2}-\gamma_{2} a_{2}^{2}\right)}\left[J_{1}\left(1+\cos \left(2 \theta_{1}\right)\right)\right.
$$$$
+J_{2} \sin \left(2 \theta_{2}\right)+J_{3} \cos \left(2 \theta_{2}\right)
$$$$
+J_{4}\left(\cos \left(\theta_{1}+\theta_{2}\right)+\cos \left(\theta_{1}-\theta_{2}\right)\right)
$$$$
+J_{5} \cos \left(2 \theta_{1}-2 \theta_{2}\right)+J_{6} \cos \left(3 \theta_{1}-3 \theta_{2}\right)
$$$$
+J_{7} \cos \left(\theta_{1}-3 \theta_{2}\right)+J_{8} \cos \left(3 \theta_{1}-\theta_{2}\right)
$$$$
\left.+J_{9} \cos \left(2 \theta_{1}-4 \theta_{2}\right)\right]+B_{1}\left(1+B_{2} \cos \left(2 \theta_{2}\right)\right) \text {, }
$$

where

$$
\begin{aligned}
& H_{1}=\frac{3}{8}\left(2+A_{2}\right) A_{1} \gamma_{1} a_{2}^{2} a_{1}^{2}, \\
& H_{2}=-\frac{1}{8}\left(4+A_{2}^{2}\right) A_{1}^{2} a_{1}^{2} \alpha_{1}, \\
& H_{3}=-\frac{3}{4}\left(1+A_{2}\right) A_{1} a_{1}^{2} a_{2}^{2} \gamma_{1}, \\
& H_{4}=-\frac{3}{16}\left(\left(2+A_{2}\right) a_{1} a_{2}^{3}+\left(6+4 A_{2}\right) a_{1}^{3} a_{2}\right) A_{1} \gamma_{1}, \\
& H_{5}=\frac{3}{8}\left(1+A_{2}\right) A_{1} a_{1}^{2} a_{2}^{2} \gamma_{1}, \\
& H_{6}=-\frac{1}{16} \gamma_{1} a_{1} A_{1} a_{2}^{3} A_{2}, \\
& H_{7}=-\frac{1}{16}\left(2+A_{2}\right) a_{1} a_{2}^{3} A_{1} \gamma_{1}, \\
& H_{8}=-\frac{3}{16}\left(a_{1} a_{2}^{3} A_{2}+\left(2+3 A_{2}\right) a_{1}^{3} a_{2}\right) A_{1} \gamma_{1}, \\
& H_{9}=\frac{3}{16} \gamma_{1} a_{1}^{2} A_{1} a_{2}^{2} A_{2},
\end{aligned}
$$




$$
\begin{aligned}
& J_{1}=-\frac{3}{8}\left(2+B_{2}\right) a_{1}^{2} a_{2}^{2} B_{1} \gamma_{2}, \\
& J_{2}=\frac{1}{12}\left(B_{2}-1\right) a_{2}^{4} B_{1}^{4} k_{3}^{3}+\left(\frac{1}{2} k_{3} \sigma-48 \alpha_{2}\right) a_{2}^{2} B_{1}^{2}, \\
& J_{3}=-\frac{3}{4}\left(B_{2}+1\right) B_{1} a_{2}^{2} a_{1}^{2} \gamma_{2}, \\
& J_{4}=\frac{3}{16}\left(\left(2+B_{2}\right) a_{2} a_{1}^{3}+\left(4 B_{2}+6\right) a_{2}^{3} a_{1}\right) \gamma_{2} B_{1}, \\
& J_{5}=-\frac{3}{8}\left(B_{2}+1\right) B_{1} a_{2}^{2} a_{1}^{2} \gamma_{2}, \\
& J_{6}=\frac{1}{16} \gamma_{2} a_{2} B_{1} a_{1}^{3} B_{2}, \\
& J_{7}=\frac{3}{16}\left(a_{2} a_{1}^{3} B_{2}+\left(3 B_{2}+2\right) a_{2}^{3} a_{1}\right) \gamma_{2} B_{1}, \\
& J_{8}=\frac{1}{16}\left(2+B_{2}\right) a_{2} a_{1}^{3} \gamma_{2} B_{1}, \\
& J_{9}=-\frac{3}{16} \gamma_{2} a_{2}^{2} B_{1} a_{1}^{2} B_{2} .
\end{aligned}
$$

(5) Letting

$$
\begin{aligned}
& \theta_{1}=\omega t+\phi_{1}, \\
& \theta_{2}=\omega t+\phi_{2},
\end{aligned}
$$

and substituting them into (24), (26), yields

$$
\begin{aligned}
& \frac{d E_{1}}{d t}=C_{1}+C_{3} \sin \left(\phi_{1}-\phi_{2}\right)+C_{4} \sin \left(2 \phi_{1}-2 \phi_{2}\right) \\
& \quad+C_{5} \sin \left(3 \phi_{1}-3 \phi_{2}\right)+\left[C_{2} \cos \left(2 \phi_{1}\right)+C_{3}\right. \\
& \quad \cdot \sin \left(\phi_{1}+\phi_{2}\right)+C_{6} \sin \left(2 \phi_{1}\right)+C_{7} \sin \left(3 \phi_{1}-\phi_{2}\right) \\
& \left.\quad+C_{8} \sin \left(\phi_{1}-3 \phi_{2}\right)+C_{9} \sin \left(4 \phi_{1}-2 \phi_{2}\right)\right] \cos (2 \omega t) \\
& +\left[-C_{2} \sin \left(2 \phi_{1}\right)+C_{3} \cos \left(\phi_{1}+\phi_{2}\right)+C_{6} \cos \left(2 \phi_{1}\right)\right. \\
& +C_{7} \cos \left(3 \phi_{1}-\phi_{2}\right)-C_{8} \cos \left(\phi_{1}-3 \phi_{2}\right)+C_{9} \\
& \left.\cdot \cos \left(4 \phi_{1}-2 \phi_{2}\right)\right] \sin (2 \omega t), \\
& \frac{d E_{2}}{d t}=D_{1}+D_{3} \sin \left(\phi_{1}-\phi_{2}\right)+D_{4} \sin \left(2 \phi_{1}-2 \phi_{2}\right) \\
& \quad+D_{5} \sin \left(3 \phi_{1}-3 \phi_{2}\right)+\left[D_{2} \cos \left(2 \phi_{1}\right)-D_{3}\right. \\
& \quad \cdot \sin \left(\phi_{1}+\phi_{2}\right)+D_{6} \sin \left(2 \phi_{2}\right)+D_{7} \sin \left(3 \phi_{1}-\phi_{2}\right) \\
& \left.+D_{8} \sin \left(\phi_{1}-3 \phi_{2}\right)+D_{9} \sin \left(2 \phi_{1}-4 \phi_{2}\right)\right] \\
& +\cos (2 \omega t)+\left[-D_{2} \sin \left(2 \phi_{1}\right)-D_{3} \cos \left(\phi_{1}+\phi_{2}\right)\right. \\
& +D_{6} \cos \left(2 \phi_{2}\right)+D_{7} \cos \left(3 \phi_{1}-\phi_{2}\right)-D_{8} \\
& \left.\cdot \cos \left(\phi_{1}-3 \phi_{2}\right)-D_{9} \cos \left(2 \phi_{1}-4 \phi_{2}\right)\right] \sin (2 \omega t)
\end{aligned}
$$

$$
\begin{aligned}
& \frac{d \theta_{1}}{d t}=\frac{1}{a_{1}^{2}\left(\beta_{1}+2 \gamma_{1} a_{1}^{2}\right)}\left\{a_{1}^{2}\left(\beta_{1}+2 \gamma_{1} a_{1}^{2}\right) A_{1}+H_{1}\right. \\
& +H_{4} \cos \left(\phi_{1}-\phi_{2}\right)+H_{5} \cos \left(2 \phi_{1}-2 \phi_{2}\right)+H_{6} \\
& \cdot \cos \left(3 \phi_{1}-3 \phi_{2}\right)+\left[H_{1} \cos \left(2 \phi_{2}\right)+H_{2} \sin \left(2 \phi_{1}\right)\right. \\
& +\left(a_{1}^{2}\left(\beta_{1}+2 \gamma_{1} a_{1}^{2}\right) A_{1} A_{2}+H_{3}\right) \cos \left(2 \phi_{1}\right) \\
& +H_{4} \cos \left(\phi_{1}+\phi_{2}\right)+H_{7} \cos \left(\phi_{1}-3 \phi_{2}\right) \\
& \left.+H_{8} \cos \left(3 \phi_{1}-\phi_{2}\right)+H_{9} \cos \left(4 \phi_{1}-2 \phi_{2}\right)\right] \\
& \cdot \cos (2 \omega t)+\left[-H_{1} \sin \left(2 \phi_{2}\right)+H_{2} \cos \left(2 \phi_{1}\right)\right. \\
& -\left(a_{1}^{2}\left(\beta_{1}+2 \gamma_{1} a_{1}^{2}\right) A_{1} A_{2}+H_{3}\right) \sin \left(2 \phi_{1}\right) \\
& -H_{4} \sin \left(\phi_{1}+\phi_{2}\right)+H_{7} \sin \left(\phi_{1}-3 \phi_{2}\right) \\
& \left.-H_{8} \sin \left(3 \phi_{1}-\phi_{2}\right)-H_{9} \sin \left(4 \phi_{1}-2 \phi_{2}\right)\right] \\
& \cdot \sin (2 \omega t)\} \text {, } \\
& \frac{d \theta_{2}}{d t}=\frac{1}{a_{2}^{2}\left(\beta_{2}-\gamma_{2} a_{2}^{2}\right)}\left\{a_{2}^{2}\left(\beta_{2}-\gamma_{2} a_{2}^{2}\right) B_{1}+J_{1}+J_{4}\right. \\
& \cdot \cos \left(\phi_{1}-\phi_{2}\right)+J_{5} \cos \left(2 \phi_{1}-2 \phi_{2}\right)+J_{6} \\
& \cdot \cos \left(3 \phi_{1}-3 \phi_{2}\right)+\left[J_{1} \cos \left(2 \phi_{1}\right)+J_{2} \sin \left(2 \phi_{2}\right)\right. \\
& +\left(a_{2}^{2}\left(\beta_{2}-\gamma_{2} a_{2}^{2}\right) B_{1} B_{2}+J_{3}\right) \cos \left(2 \phi_{2}\right) \\
& +J_{4} \cos \left(\phi_{1}+\phi_{2}\right)+J_{7} \cos \left(\phi_{1}-3 \phi_{2}\right) \\
& \left.+J_{8} \cos \left(3 \phi_{1}-\phi_{2}\right)+J_{9} \cos \left(2 \phi_{1}-4 \phi_{2}\right)\right] \cos (2 \omega t) \\
& +\left[-J_{1} \sin \left(2 \phi_{1}\right)+J_{2} \cos \left(2 \phi_{2}\right)\right. \\
& -\left(a_{2}^{2}\left(\beta_{2}-\gamma_{2} a_{2}^{2}\right) B_{1} B_{2}+J_{3}\right) \sin \left(2 \phi_{2}\right) \\
& -J_{4} \sin \left(\phi_{1}+\phi_{2}\right)+J_{7} \sin \left(\phi_{1}-3 \phi_{2}\right) \\
& \left.-J_{8} \sin \left(3 \phi_{1}-\phi_{2}\right)-J_{9} \sin \left(2 \phi_{1}-4 \phi_{2}\right)\right] \\
& \cdot \sin (2 \omega t)\} \text {. }
\end{aligned}
$$

If there exists a periodic solution with period $2 \pi / \omega$ in (3), then the energy of the system should remain the same at initial and finial positions in one period. Integrating both sides of (29) in one period (from $t=\Delta$ to $t=\Delta+2 \pi / \omega$ ) leads to

$$
\begin{aligned}
& \left(E_{1}\right)_{2 \pi / \omega+\Delta}-\left(E_{1}\right)_{\Delta}=C_{1}=-\frac{1}{4}\left[\left(A_{2}-1\right)^{2}-1\right] \\
& \cdot A_{1}^{2} a_{1}^{2} \alpha_{1}<0, \\
& \left(E_{2}\right)_{2 \pi / \omega+\Delta}-\left(E_{2}\right)_{\Delta}=D_{1}=\frac{B_{1}^{2} a_{2}^{2}}{24}\left[\left(8 B_{2}-3\right) B_{1}^{2} a_{2}^{2} k_{3}^{3} \sigma\right. \\
& \left.\quad-12\left(B_{2}-1\right)\left(k_{3} \sigma-\alpha_{2}\right)\right] .
\end{aligned}
$$



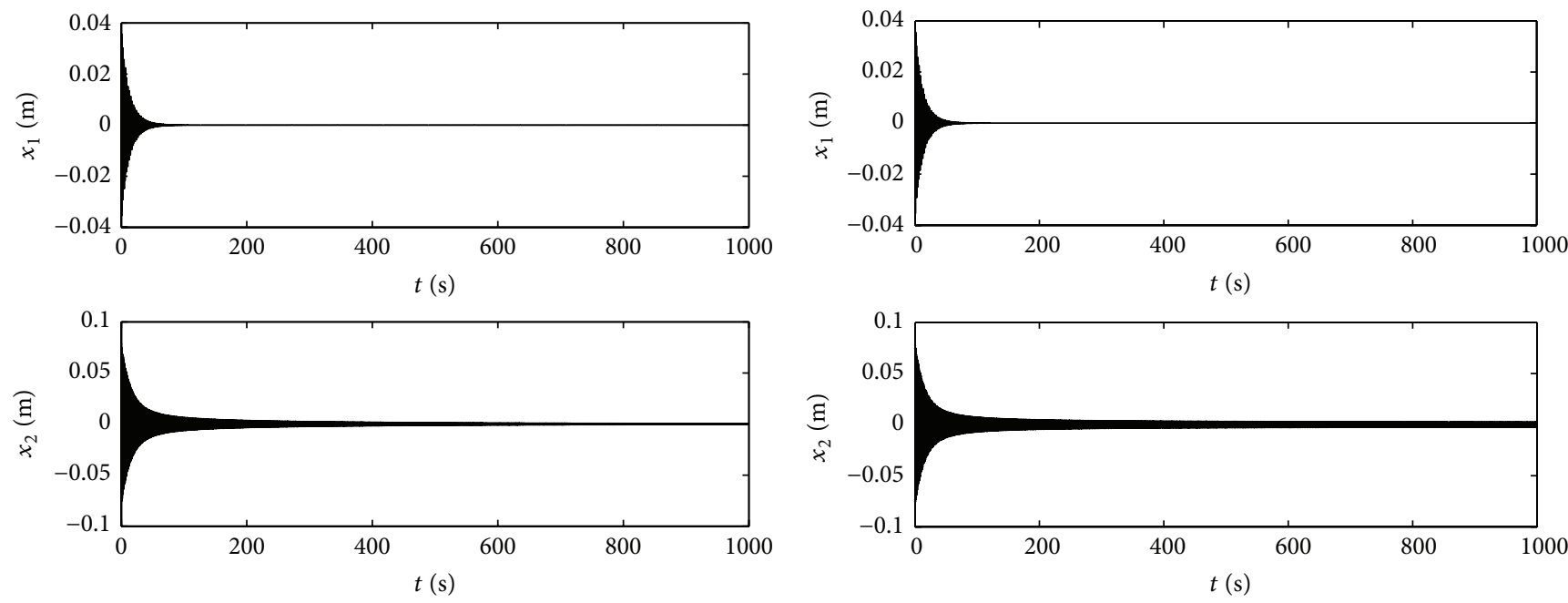

(a)

(b)

FIGURE 2: Direct numerical simulations of (3) for (a) $n=112$ and (b) $n=118$.

Inspection of the first equation in (31) shows that the vibration energy of cables decreases in one period. Therefore, cables cannot vibrate periodically when the deck is subjected to the crowd excitation. From the second equation in (31), if $k_{3} \sigma-\alpha_{2}<0$, then $D_{1}<0$. At this point, the deck has an attenuate vibration. If $k_{3} \sigma-\alpha_{2}>0, D_{1}=0$ may hold. Letting $D_{1}=0$ in the second equation in (31) and ignoring the sixth or more power of $a_{2}$ (since $a_{2} \ll 1$ according to its physical meaning), one obtains

$$
7 \gamma_{2} a_{2}^{4} \beta_{2} \sigma k_{3}^{3}-3\left(5 \delta \gamma_{2}+2 \beta_{2}^{2} \sigma k_{3}^{3}\right) a_{2}^{2}+24 \delta \beta_{2}=0
$$

where $\delta=k_{3} \sigma-\alpha_{2}$. Solving (32) yields

$$
\begin{aligned}
& a_{2}^{*} \\
& =\sqrt{\frac{6 \sigma k_{3}^{3} \beta_{2}^{2}+15 \gamma_{2} \delta-\sqrt{12 \sigma k_{3}^{3} \beta_{2}^{2}\left(3 \sigma k_{3}^{3} \beta_{2}^{2}-41 \gamma_{2} \delta\right)+225 \gamma_{2}^{2} \delta^{2}}}{14 \sigma \gamma_{2} k_{3}^{3} \beta_{2}}} \\
& =2 \sqrt{2} \sqrt{\frac{\beta_{2} \delta}{2 \sigma k_{3}^{3} \beta_{2}^{2}+5 \gamma_{2} \delta}} .
\end{aligned}
$$

For clarification, the amplitude can be rewritten as

$$
a_{2}^{*}=4 \sqrt{\frac{m_{2} k\left(k_{3} \gamma-c_{2}\right)}{4 \gamma k_{3}^{3} k^{2}+5\left(E A / 2 L^{3}\right) m_{2}\left(k_{3} \gamma-c_{2}\right)}},
$$

where $\gamma=k_{1} k_{2} G(f) M_{p} g$ and $k_{3} \gamma-c_{2}>0$.

(6) The first-order approximation solution of (3) is expressed by

$$
\begin{aligned}
x_{2}(t) & =a_{2}^{*} \cos (\omega t) \\
& =a_{2}^{*} \cos \left(\sqrt{\frac{1}{m_{2}}\left[k-\frac{3}{8} \frac{E A}{L^{3}}\left(a_{2}^{*}\right)^{2}\right] t}\right),
\end{aligned}
$$

\section{Numerical Simulations and Discussion}

In this section we will verify the validity of our analytical solutions obtained in the last section by comparing the results based on (35) with that derived by direct numerical simulations for (3). The following parameters of deck and pedestrians are taken to calculate the periodic solutions in (3):

$$
\begin{aligned}
m_{2} & =2.1401 \times 10^{5}(\mathrm{~kg}), \\
k & =7.307361 \times 10^{6}\left(\mathrm{~kg} / \mathrm{s}^{2}\right), \\
c_{2} & =2.8262 \times 10^{4}(\mathrm{~kg} / \mathrm{s}), \\
G(f) & =1.0, \\
k_{1} & =0.0987, \\
k_{2} & =0.2, \\
k_{3} & =18 .
\end{aligned}
$$

In fact, above values of parameters are used in $[17,19]$ for the first lateral model of the T-bridge in Japan. Furthermore, the weight of a single person on the bridge is assumed to be $70(\mathrm{~kg})$; the gravity acceleration $g=9.8\left(\mathrm{~m} / \mathrm{s}^{2}\right)$. The modal mass of pedestrians $M_{p} g$ can be given by $M_{p} g=n \times 70 \times 9.8$, where $n$ is the number of the pedestrians on the bridge. Additionally, according to [19], the average length of the cables is taken as $L=60(\mathrm{~m})$. However, other parameters of the cables, such as stiffness, damping coefficient, and mass, are not provided in [19]. In order to compare the theoretical results of T-bridge between our cable-stayed bridge model and Nakamura's model, following parameters of cables are assumed in the numerical simulations:

$$
\begin{aligned}
& m_{1}=2.1401 \times 10^{3}(\mathrm{~kg}), \\
& E A=1.68 \times 10^{10}(\mathrm{~N}),
\end{aligned}
$$




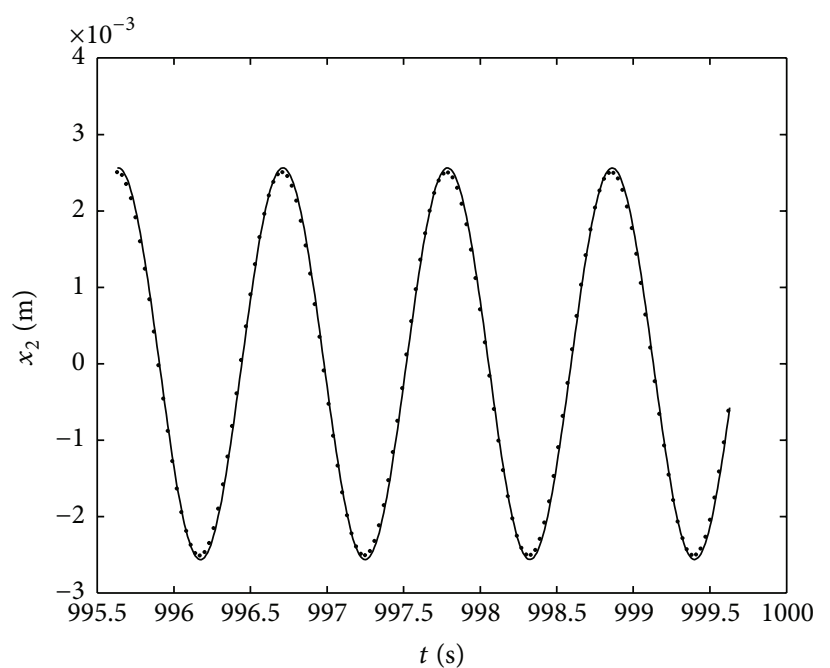

- Numerical results

- Analytical results

(a)

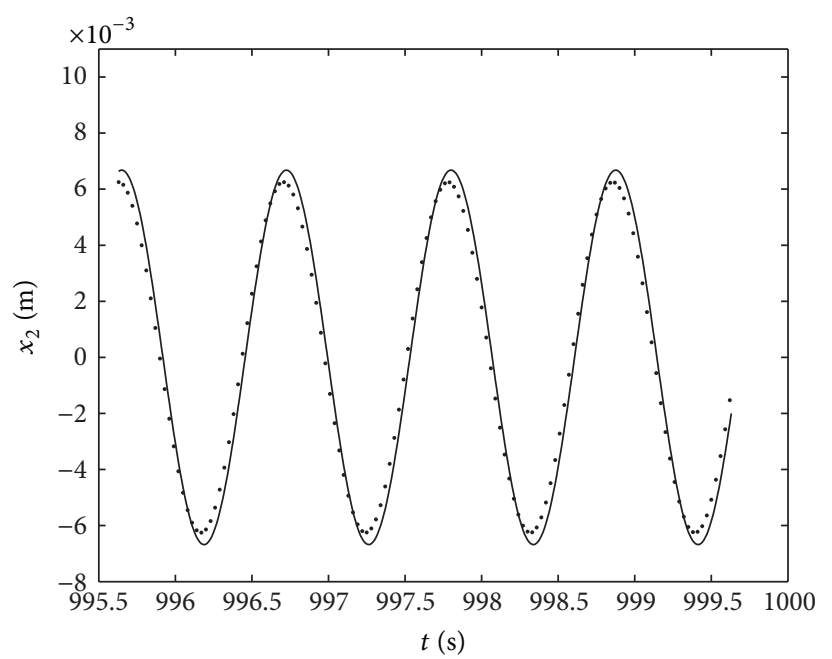

- Numerical results

- Analytical results

(c)

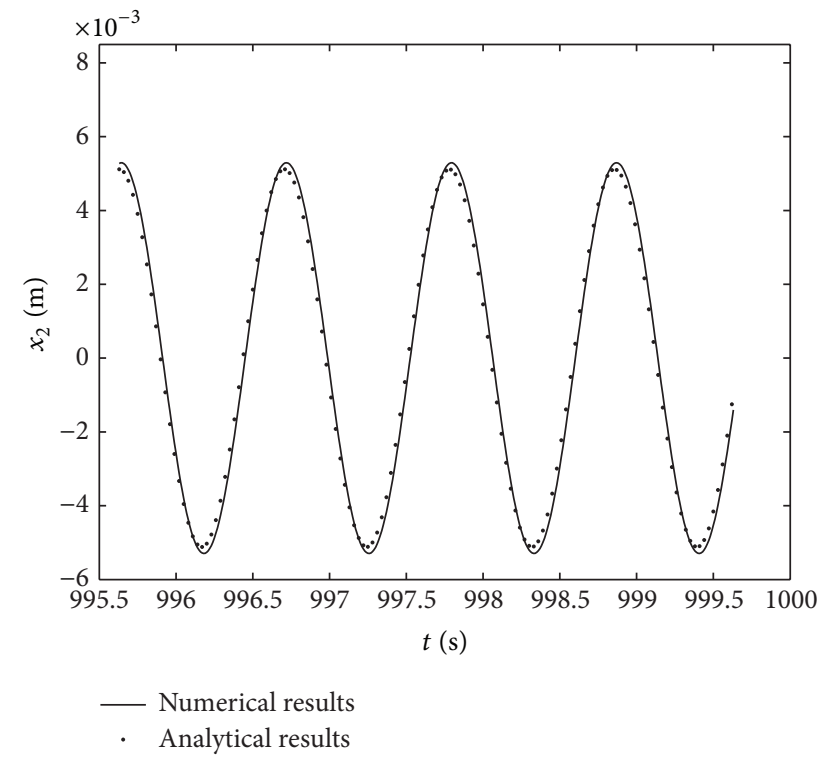

(b)

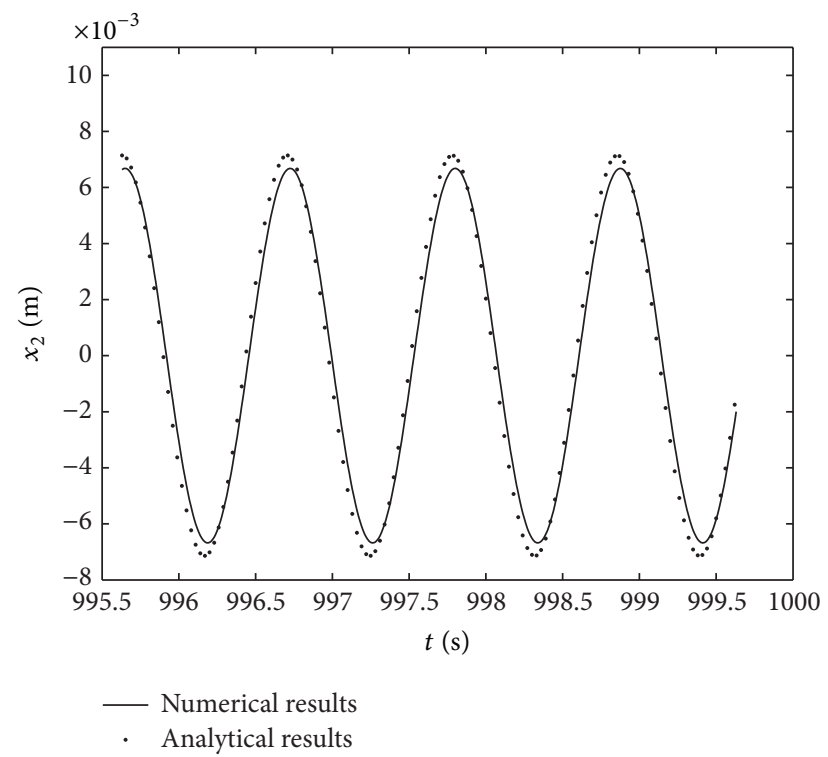

(d)

FIGURE 3: Comparison between the first-order approximation solution equation (35) and direct numerical results of (3) for the cases of (a) $n=118$, (b) $n=125$, (c) $n=130$, and (d) $n=135$.

$$
\begin{aligned}
c_{1} & =2.8262 \times 10^{2}(\mathrm{~kg} / \mathrm{s}), \\
T_{0} & =7.24 \times 10^{5}(\mathrm{~N}) .
\end{aligned}
$$

Then we have

$$
\begin{aligned}
& \alpha_{1}=0.1321(1 / \mathrm{s}), \\
& \alpha_{2}=0.1321(1 / \mathrm{s}), \\
& \beta_{1}=67.6604\left(1 / \mathrm{s}^{2}\right), \\
& \beta_{2}=34.1450\left(1 / \mathrm{s}^{2}\right),
\end{aligned}
$$

$$
\begin{aligned}
& \gamma_{1}=3.9251 \times 10^{3}\left(1 / \mathrm{s}^{2}\right), \\
& \gamma_{2}=39.2505\left(1 / \mathrm{s}^{2}\right) .
\end{aligned}
$$

Regarding the number $n$ as the bifurcation parameter, one has

$$
\begin{aligned}
& \sigma=\frac{k_{1} k_{2} G(f) M_{p} g}{m_{2}}=0.000063275734785 n, \\
& \delta=k_{3} \sigma-\alpha_{2}=0.00113896 n-0.13201 .
\end{aligned}
$$

From the analysis in Section 3, $\delta<0$ means the attenuation of the lateral vibration of the bridges, while $\delta>0$ means the 


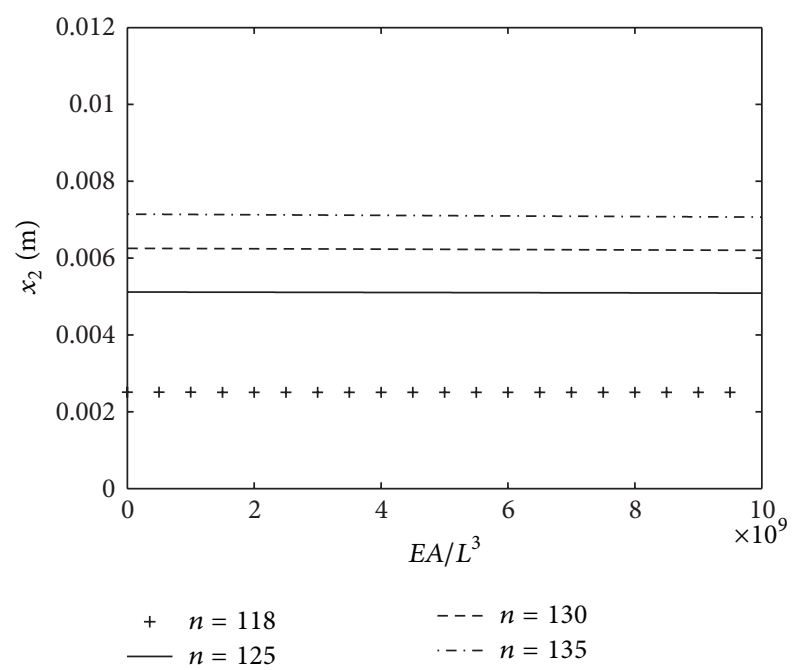

(a) $x_{2}$ versus $E A / L^{3}$

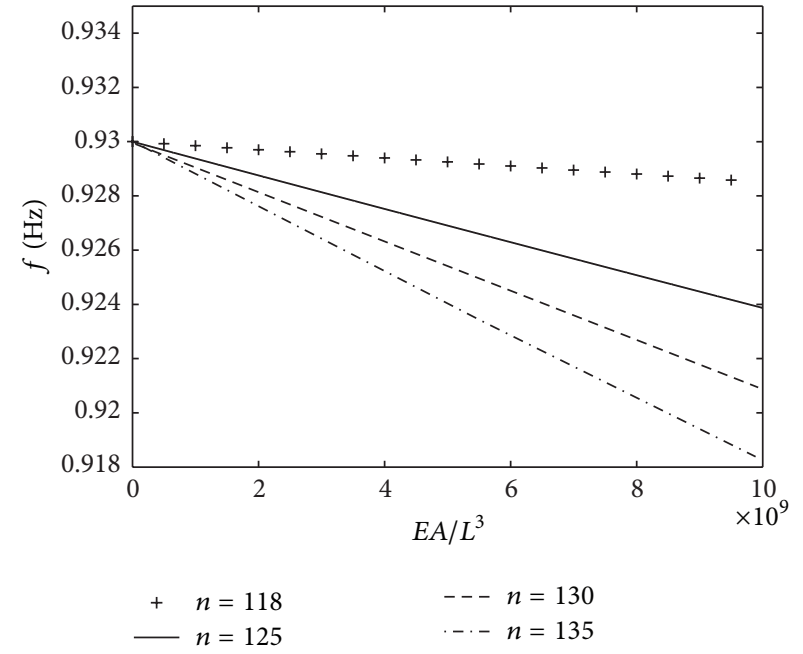

(b) $f$ versus $E A / L^{3}$

Figure 4: The (a) amplitude $\left(x_{2}\right)$ and (b) frequency $(f)$ of deck always decrease with $E A / L^{3}$ which is increased based on (35) for different numbers of pedestrians on the bridge.

occurrence of the lateral vibration of the bridge. No matter what the motion of the deck is, the cables vibration always is attenuated. Thus, $n=115$ is the critical point corresponding to the lateral vibration of the deck. The time history curves of displacements of the cables and deck for cases of $n=112$ and $n=118$ are presented in Figure 2 by direct numerical simulations for (3), which demonstrates the correctness of our analysis in Section 3. The initial condition in numerical simulations is given by $x_{1}(0)=0, \dot{x}_{1}(0)=0, x_{2}(0)=0.08$, and $\dot{x}_{2}(0)=0$.

In the following, the analytical results based on (35) are compared with that obtained by direct numerical simulations of (3) for cases of $n=118,125,130$, and 135, respectively, which are shown in Figure 3. It shows good agreement between the two approaches for $n$ near bifurcation point $n=$ 115. However, the precision of the first-order approximation solution is lost when $n$ is considerably larger than the value of the bifurcation point. In this case, second-order or more order approximation is needed to be calculated for higher precision of the analytical solution.

Since the validity of (35) has been verified, in the rest of the section we will consider the behavior of system equation (3) on varying the parameters based on (35). From (35), the amplitude of the lateral vibration of a cable-stayed bridge under crowd excitation has nothing to do with the mass, tension, and damping coefficient of the cables. But the existence of the cables can decrease the amplitude of the deck since the term of $E A / L^{3}$ appears in the denominator. This means that the theoretical results of the vibration amplitude of the deck derived by using (3) are less than that obtained by using Nakamura's model in [19]. For the first lateral model of the T-bridge, the theoretical result is higher by $49 \%$ than the measured data. Nakamura considered that the reason is the uncertainty of measurements of parameters such as modal mass, stiffness, and damping coefficient of the deck. However, only using a dating error is difficult to explain such a big difference between theoretical results and measured data. Equation (35) shows that neglecting the structural type of the cable-stayed bridge may be an important cause of the difference between theoretical and measured results. Furthermore, from (35) the frequency of the lateral vibration of the deck also decreases due to the existence of the cables. The curves about the change of the amplitude and frequency of the deck with the term of $E A / L^{3}$ for different numbers of pedestrians are given in Figure 4 based on (35), in which the parameters of deck and pedestrians presented in (36) are adopted. From Figure 4, the more the pedestrians on the bridge are the faster the vibrational frequency decreases with $E A / L^{3}$ which is increased.

Figures 5 and 6 present the curves about the change of the amplitude and frequency of the deck with the terms of $k_{2}$, $k_{3}$, respectively, for different numbers of pedestrians based on (35), in which all other parameters of deck and cables are the same as that presented in (36) and (37). It can be seen from Figures 5 and 6 that changes of $k_{2}, k_{3}$ have significant effect on the amplitude of the deck and, however, almost have nothing to do with its vibrational frequency.

\section{Conclusions}

In this paper, the lateral vibrations of a cable-stayed bridge under crowd excitation are investigated theoretically and numerically. In our study, the cable-stayed bridge is simplified as a string and a rigid block, and the pedestrian-induced force model is satisfied with Nakamura's assumption. The governing equation of our model is established by taking the geometric nonlinear property of the cables into account. Center manifold theory is employed to determine the critical condition that periodic vibrations of the bridge occur. Then first-order approximation solution of periodic vibrations of the deck is calculated by using the energy method, the validity of which is verified by using direct numerical simulations. 


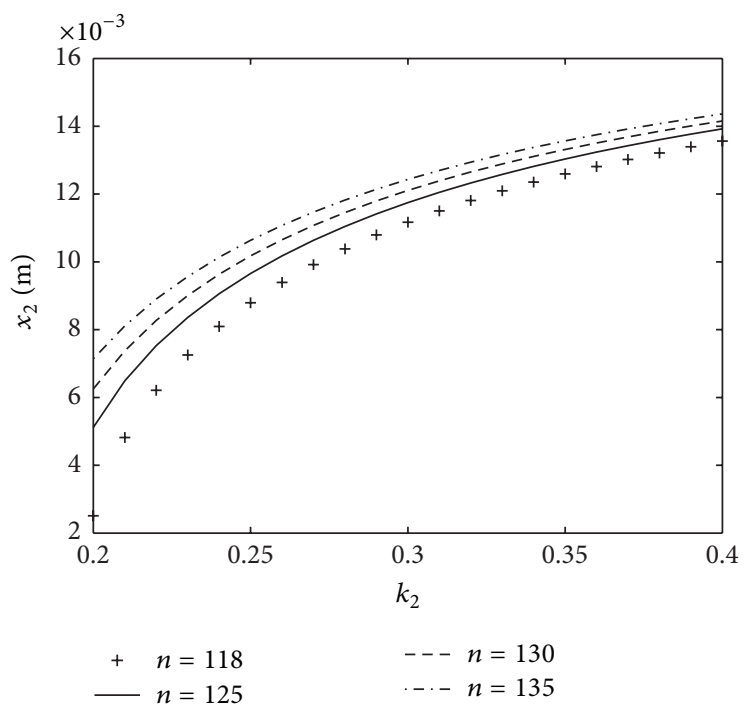

(a) $x_{2}$ versus $k_{2}$

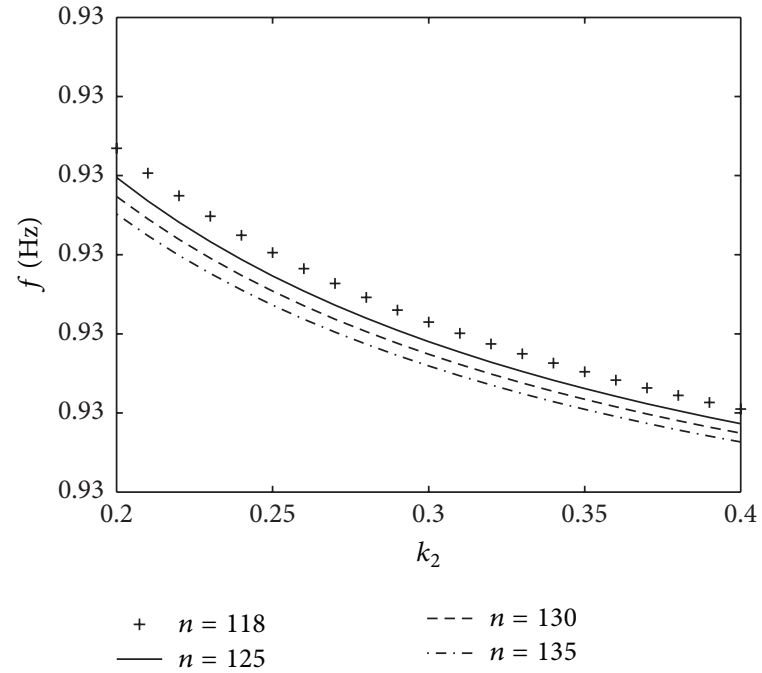

(b) $f$ versus $k_{2}$

Figure 5: The curves of (a) amplitude $\left(x_{2}\right)$ and (b) frequency $(f)$ of deck change with $k_{2}$ based on (35) for different numbers of pedestrians on the bridge.

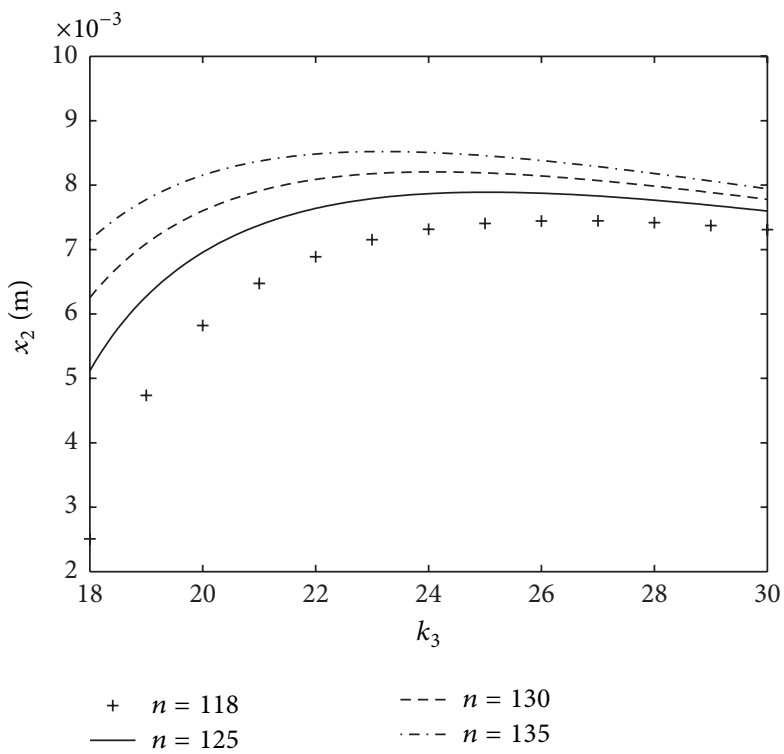

(a) $x_{2}$ versus $k_{3}$

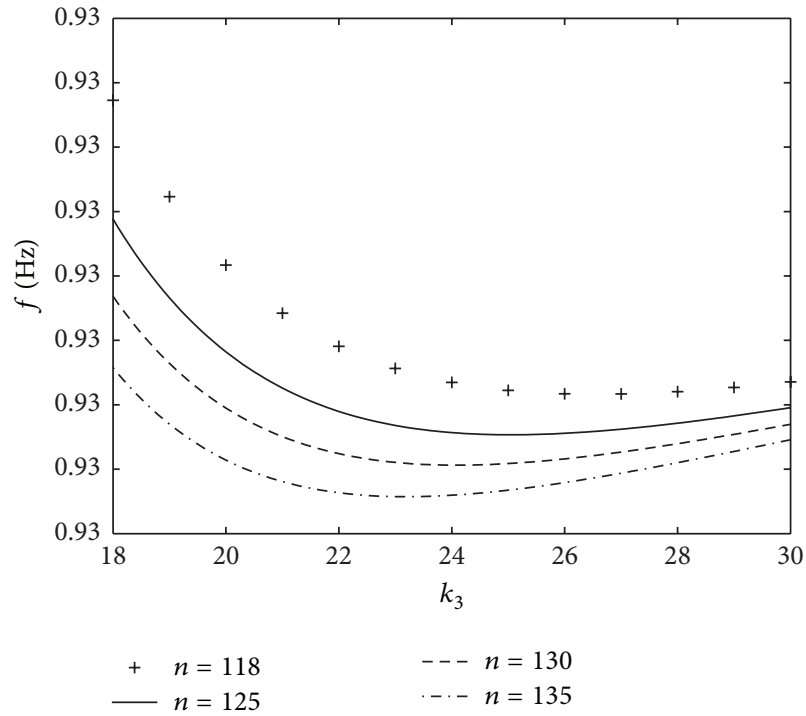

(b) $f$ versus $k_{3}$

Figure 6: The curves of (a) amplitude $\left(x_{2}\right)$ and (b) frequency $(f)$ of deck change with $k_{3}$ based on (35) for different numbers of pedestrians on the bridge.

Our analysis shows that cables can have no periodic vibration even if the deck loses its stability and begins to sway, but cables can affect the amplitude and frequency of the lateral vibration of the deck. From the first-order approximation solution equations (34) and (35), the mass, damping coefficient, and tension of the cables have no effect on the lateral vibrations of the deck. However, existence of the cables always reduces the amplitude and frequency of the lateral vibrations of the deck. With the bifurcation parameter far away from the bifurcation point, the cables have a growing influence on both amplitude and frequency of the deck. Our analysis results may be used to explain why the measured results for T-bridge (a cable-stayed bridge) derived by Nakamura and Kawasaki [19] are much less than their theoretical results calculated by regarding the T-bridge as a single-degree-of-freedom system. This indicates that the structure types of the footbridges cannot be easily ignored in the study of pedestrian-footbridge interaction. 


\section{Conflict of Interests}

The authors declare that there is no conflict of interests regarding the publication of this paper.

\section{Authors' Contribution}

All authors carried out the proofreading of the paper. All authors conceived of the study and participated in its design and coordination. All authors read and approved the final paper.

\section{Acknowledgments}

This work was supported by the National Natural Science Foundation of China (Grant nos. 11472160), the State Key Program of National Natural Science of China (Grant no. 11032009), and Ph.D. start-up fund of Shanghai Publishing and Printing College 95-A (1030114203). Furthermore, the authors appreciate very much the helpful advice of the reviewers.

\section{References}

[1] P. Dallard, A. J. Fitzpatrick, A. Flint et al., "The London millennium footbridge," Structural Engineer, vol. 79, no. 22, pp. 1733, 2001.

[2] P. Dallard, T. Fitzpatrick, A. Flint et al., "London millennium bridge: pedestrian-induced lateral vibration," Journal of Bridge Engineering, vol. 6, no. 6, pp. 412-417, 2001.

[3] Y. Fujino, B. M. Pacheco, S.-I. Nakamura, and P. Warnitchai, "Synchronization of human walking observed during lateral vibration of a congested pedestrian bridge," Earthquake Engineering and Structural Dynamics, vol. 22, no. 9, pp. 741-758, 1993.

[4] S.-I. Nakamura, "Model for lateral excitation of footbridges by synchronous walking," Journal of Structural Engineering, vol. 130, no. 1, pp. 32-37, 2004.

[5] Y. Fujino, P. Warnitchai, and B. M. Pacheco, "An experimental and analytical study of autoparametric resonance in a $3 \mathrm{DOF}$ model of cable-stayed-beam," Nonlinear Dynamics, vol. 4, no. 2, pp. 111-138, 1993.

[6] H. Bachmann, A. J. Pretlove, and H. Rainer, "Dynamic forces from rhythmical human body motions," in Vibration Problems in Structure: Practical Guidelines, Birkhauser, Basel, Switzerland, 1995.

[7] A. N. Blekherman, "Autoparametric resonance in a pedestrian steel arch bridge: solferino bridge, Paris," Journal of Bridge Engineering, vol. 12, no. 6, pp. 669-676, 2007.

[8] T. M. Roberts, "Lateral pedestrian excitation of footbridges," Journal of Bridge Engineering, vol. 10, no. 1, pp. 107-112, 2005.

[9] S. H. Strogatz, D. M. Abrams, A. McRobie, B. Eckhardt, and E. Ott, "Crowd synchrony on the Millennium Bridge," Nature, vol. 438, no. 7064, pp. 43-44, 2005.

[10] F. Venuti, L. Bruno, and N. Bellomo, "Crowd dynamics on a moving platform: mathematical modelling and application to lively footbridges," Mathematical and Computer Modelling, vol. 45, no. 3-4, pp. 252-269, 2007.
[11] S. Lenci and L. Marcheggiani, "Critical threshold and underlying dynamical phenomena in pedestrian-induced lateral vibrations of footbridges," Journal of Mechanics of Materials and Structures, vol. 6, no. 7-8, pp. 1031-1051, 2012.

[12] G. Piccardo and F. Tubino, "Parametric resonance of flexible footbridges under crowd-induced lateral excitation," Journal of Sound and Vibration, vol. 311, no. 1-2, pp. 353-371, 2008.

[13] A. McRobie, G. Morgenthal, J. Lasenby, and M. Ringer, "Section model tests on human-structure lock-in," Proceedings of the ICE-Bridge Engineering, vol. 156, no. 2, pp. 71-79, 2003.

[14] D. Zhou and T. J. Ji, "Dynamic characteristics of a generalised suspension system," International Journal of Mechanical Sciences, vol. 50, no. 1, pp. 30-42, 2008.

[15] C. Geurts, T. Vrouwenvelder, P. V. Staalduinen, and J. Reusink, "Numerical modeling of rain-wind-indued vibration: erasmus Bridge, Rotterdam," Structural Engineering International, vol. 8, no. 2, pp. 129-135, 1998.

[16] J. L. Lilien and A. Pinto Da Costa, "Vibration amplitudes caused by parametric excitation of cable stayed structures," Journal of Sound and Vibration, vol. 174, no. 1, pp. 69-90, 1994.

[17] B. Zhen, W. P. Xie, and J. Xu, "Nonlinear analysis for the lateral vibration of footbridges induced by pedestrians," Journal of Bridge Engineering, vol. 18, no. 2, pp. 122-130, 2013.

[18] L. Li and Y. Hongling, "Energy method for computing periodic solutions of strongly nonlinear autonomous systems with multidegree-of-freedom," Nonlinear Dynamics, vol. 31, no. 1, pp. 2347, 2003.

[19] S. Nakamura and T. Kawasaki, "A method for predicting the lateral girder response of footbridges induced by pedestrians," Journal of Constructional Steel Research, vol. 65, no. 8-9, pp. 1705-1711, 2009. 


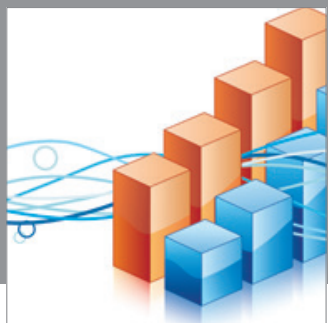

Advances in

Operations Research

mansans

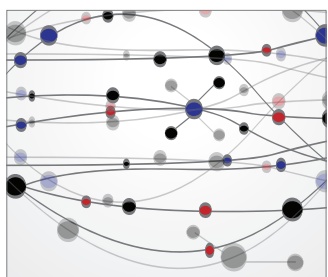

The Scientific World Journal
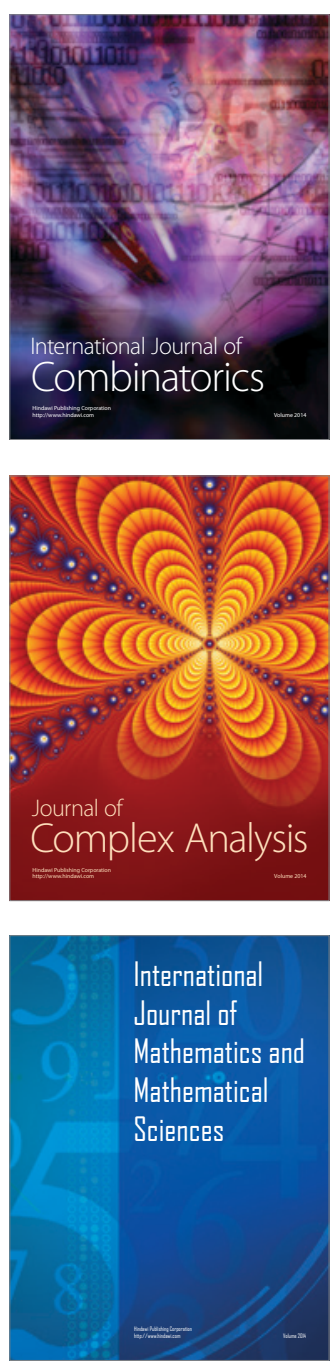
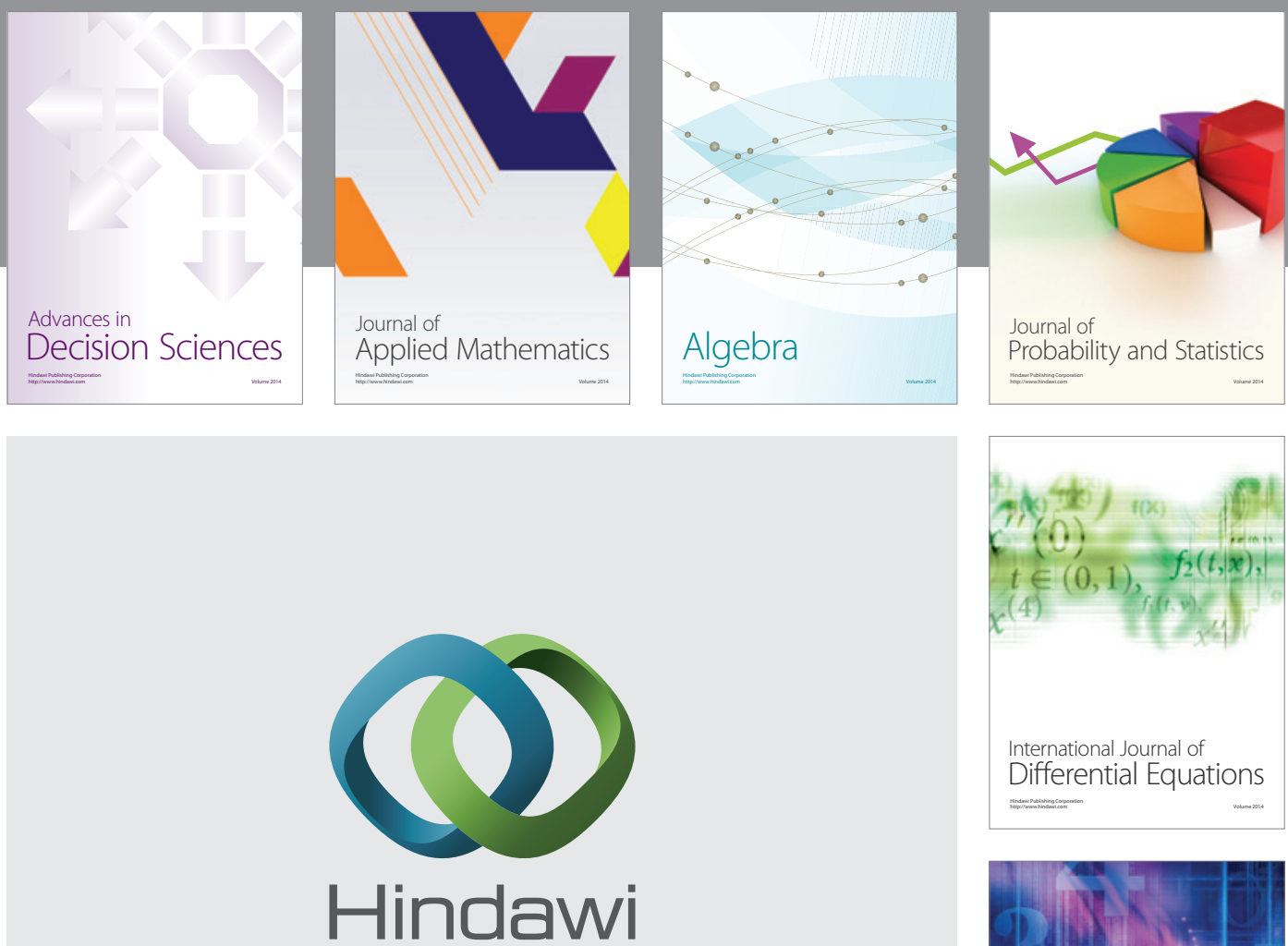

Submit your manuscripts at http://www.hindawi.com
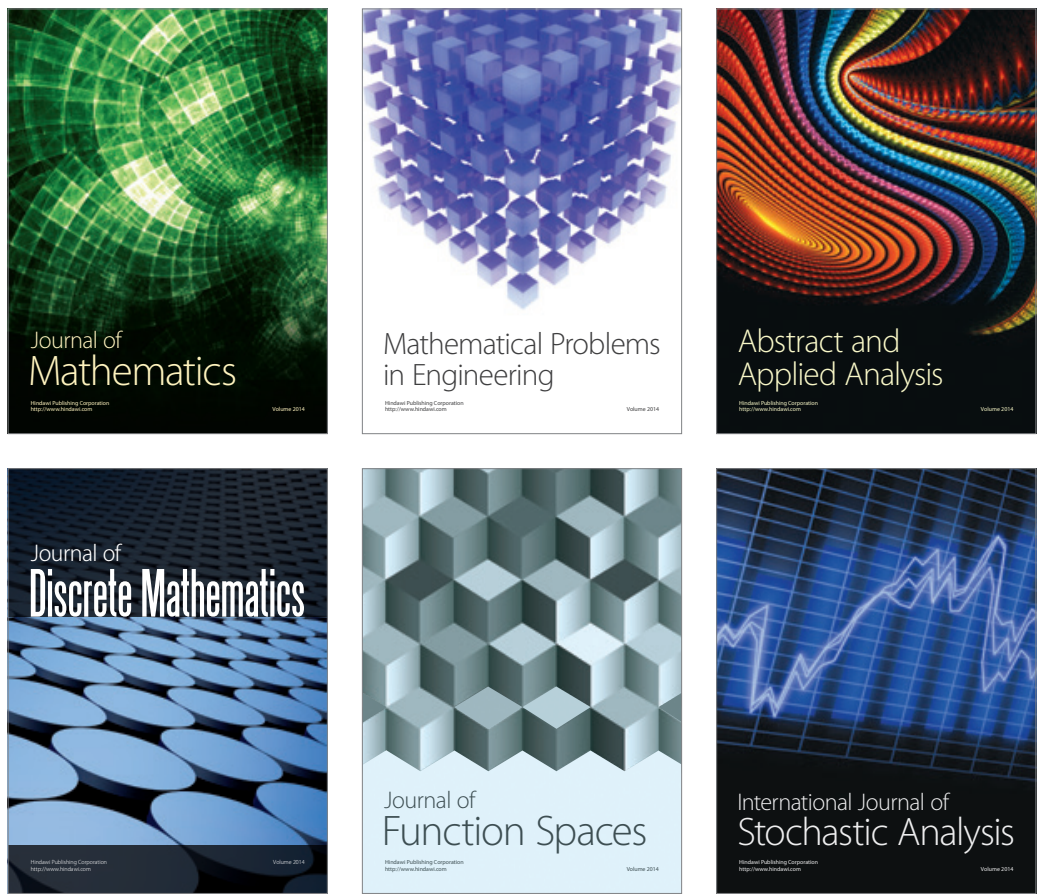

Journal of

Function Spaces

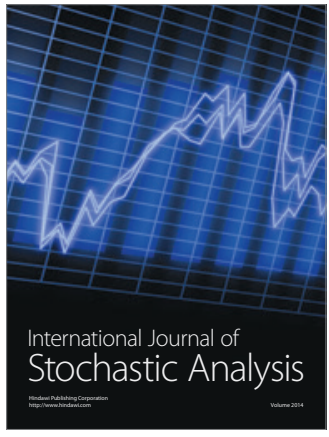

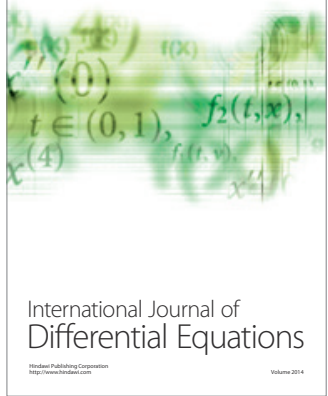
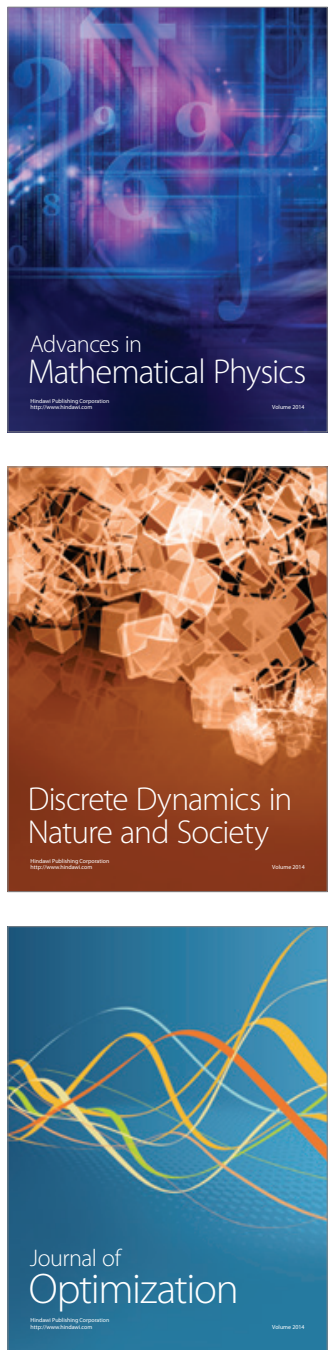